\title{
Successful pregnancy after complex treatment of giant uterine myoma
}

\begin{abstract}
Introduction: It is known that uterine leiomyoma (LM) may relapse following myomectomy performed by different access techniques in $5-67 \%$ of patients, and the incidence of repeat surgery is $3.2-30 \%$.

Material and methods: Clinical observation: female patient, 36years of age; pregnancies: 0 ; menstruation since 12 years of age, $6 / 25-27$, moderate, painless; sex life since 16years of age; contraception: condoms; gynecological disorders: denies; family history: negative; LM since 2010, gradually growing; diagnosis: LM 18 weeks, infertility 1 ; laparotomic myomectomy ( 6 fibroids removed). The follow-up examination six months later identified the relapse of LM (sizes: 55x47x68; 49x47 with deformed uterine cavity, complaints of heavy periods, fatigue, $\mathrm{Hb} 76 \mathrm{~g} / \mathrm{L}$ ). Diagnosis: Recurrent LM following myomectomy, menorrhagia, iron deficiency anemia 2. Hysterectomy was proposed. The patient strongly refused the radical treatment owing to reproductive plans. Decision: Repeat myomectomy following a course of pre-operative treatment. Prescription: Ulipristal acetate (Esmya) 1 tablet once daily + Sorbifer Durules 1 tablet twice daily for 84 days. 3months later, it was reported that UA caused amenorrhea within 7days after drug prescription, and the use of UA \& Sorbifer Durules allowed the restoration of hemoglobin level (up to $110 \mathrm{~g} / \mathrm{L}$ ) within 3 months and the decrease in the risks associated with planned surgical intervention as well. LM reduced in size by $30 \%$. 2weeks following this treatment, the laparotomic myommyometrectomy was performed; blood loss was lower than $150 \mathrm{~mL}$ owing to the use of our method of atraumatic controlled vasocompression.
\end{abstract}

Results: Opening of pseudocapsule of leiomyoma was uneventful. The post-operative period was uneventful. 3 days later, the patient was discharged in generally satisfactory condition. Histological examination result: Simple LM with apoptotic figures moderately developed extracellular matrix, microvascular network not pronounced, normal myometrium layer and serosal covering. Leiomyoma is presented with differently organized areas without signs of atypical mitosis or leiomyocyte atypia and necrotic foci. Pseudocapsule is pronounced. UA (Esmya) $5 \mathrm{mg} /$ day was started from the first day of the patient's new menstruation once per 28days N3. US examination in 3 months: uterus $4.9 \times 4.9 \times 5.6$; M-echo $3 \mathrm{~mm}$, endometrium is linear, uterine cavity outlines are smooth and sharp. There are 5 follicles (ovaries) in the section. The combined oral contraceptive Siluet was prescribed for 6 months following restoration of menstruation. After 6months, combined oral contraceptives were stopped. Reproduction plans. In 3months: 10day-delay of menstruation, positive pregnancy test. US examination in 3 weeks: Normally progressing pregnancy $6 \mathrm{GW}$. Following a US examination at $11 \mathrm{GW}, 17 \mathrm{GW}, 34 \mathrm{GW}$ : Normally progressing pregnancy. At $38-$ 39GW: Successful delivery through C-section with live male fetus $(3,050 \mathrm{~g})$.

Conclusion: To create conditions for organ-preserving treatment and reduction of surgical intervention risks, a differential approach combining medical and surgical treatment for recurrent LM is required. The most reasonable therapeutic component in this respect may be application of selective PR modulator, UA (Esmya) \& Fe++ (Sorbifer Durules), as an important part of combined treatment of patients with uterine fibroids.

Keywords: giant uterine myoma, uterine fibroids, myomectomy, GNRH agonists
Volume 3 Issue 4 - 2017

Tikhomirov AL

Moscow State Medico-stomatological University, Russia

Correspondence: Tikhomirov AL, professor, Moscow State Medico-stomatological University, Russia, Email tikhomiroval@yandex.ru

Received: November 18, 2017 | Published: December 27, 2017
Abbreviations: UA, ulipristal acetate; IDA, iron deficiency anemia; myoma EMM, embolization of uterine myoma; SMRP, selective modulator of progesterone receptors

\section{Introduction}

This report describes a progressing pregnancy after the complex treatment of giant uterine fibroids with the use of ulipristal acetate (UA). A young, unfertilized patient (27years old) with reproductive plans, when examining menometrorrhagia and iron deficiency anemia
(IDA), found a fast growing uterine myoma, corresponding to 30 weeks of pregnancy. In order to reduce the size of uterine fibroids, hemostasis and correction of IDA before conservative myomectomy, the patient underwent embolization of uterine myoma (EMM) and a 3-month course of UA therapy, since surgical organ-preserving treatment of patients with giant-sized uterine myoma is classified as technically complex operations and has a high risk of intra- and postoperative complications. This observation demonstrates modern tactics of managing patients with uterine myoma, implying a differentiated 
approach, a combination of medical and surgical treatment of uterine fibroids.

In women of reproductive age, uterine fibroids remain the most common neoplasm with indication for hysterectomy. Rapid growth of uterine fibroids is observed in approximately $20-40 \%$ of women of childbearing age. ${ }^{1}$ The progression of symptoms and the lack of a sufficient effect of pharmacotherapy until recently, necessitated the surgical intervention. Myomectomy is an effective method of treating uterine fibroids in women who want to maintain and restore their reproductive function. In the last two decades, the number of developments has increased in the use of less invasive and sparing operations in such patients with uterine myoma - laparoscopic or hysteroscopic myomectomy and image-guided methods such as uterine artery embolization (EMA). Introduced into clinical practice in the late 1990s, EMA as an alternative to hysterectomy has proven to be effective, minimally invasive and low risk of complications in the treatment of women with uterine myoma. However, patients with large uterine myoma are candidates for abdominal myomectomy.

Among the most recent available therapeutic options, Ulipristal acetate (UA) has proved to be the most effective as a preoperative supplement. UA is a selective modulator of progesterone receptors (SMRP), registered for the treatment of uterine fibroids. The efficacy of UA at a dose of $5 \mathrm{mg}$ per day for 13 weeks was demonstrated with respect to a decrease in the intensity of bleeding and a decrease in the size of uterine fibroids. It is also even more effective, as do GnRH agonists (aHnRH), with regard to reducing uterine bleeding, but it results in significantly less frequent side effects. ${ }^{2}$ Data on benign histological changes in the endometrium (PAEC) caused by ulceration of acetate appear to be encouraging, as these changes tend to spontaneously resolve within a few months after the end of the 13week treatment period. ${ }^{3,4}$

\section{Material and methods}

In a young patient, 27years of age with reproductive plans, a rapid growth of uterine fibroids, corresponding to 30 weeks of gestation, was detected in the course of the examination for menometrorrhagia. There were no pregnancies. Menses - profuse, painful. The last visit to the gynecologist was in the spring of 2012, then the uterine fibroids were not. Since November 2012 marked a gradual increase in the abdomen, menstruation became more abundant and prolonged, there was a weakness. 04/06/2013 The patient turned to the gynecologist at the place of residence in the city of Yaroslavl. A diagnosis was made: "Myoma of the uterus is 30 weeks old with an intramural node of $22 \mathrm{~cm}$ in diameter, menometrorrhagia, an IDA of the 2nd stage" and a hysterectomy is proposed for the rapid growth, the giant size of the uterine myoma. From the radical treatment the patient categorically refused.

The patient was interested in pregnancy and 10.04.2013. Asked for a consultation in our clinic. In order to prepare for a conservative myomectomy on $04 / 20 / 2013$. The patient underwent embolization of uterine myoma.

The use of aGnRH instead of UAE was not justified because of the size of uterine fibroids, the young age of the patient, and the risk of difficulties in subsequent enucleation of the myomatous node. The drug Esmya (UA) at that time was still inaccessible in Russia. After embolization (May-June 2013), there was a reduction in the duration and volume of menstrual blood loss, a decrease in the size of the abdomen.
In September 2013 the patient was re-consulted in our clinic. There was a reduction in the size of the node to $15 \times 11 \times 13 \mathrm{~cm}$, but the uterine cavity was sharply deformed. Persistent menstruation persisted, iron deficiency anemia of moderate severity was diagnosed, hemoglobin - $77 \mathrm{~g} / 1$. To reduce the amount of menstrual bleeding, correction of anemia and preparation for surgery, UA was prescribed $5 \mathrm{mg}$ per day for 3months + Fe Sorbifer Durules. 12/28/2013 it is fixed that UA up to 7 days after the appointment caused amenorrhea, after 3 months allowed to restore hemoglobin $(110 \mathrm{~g} / 1)$, and thereby reduce the risks associated with the upcoming surgical treatment. After a pronounced decrease in the size of the node after embolization of the uterine myoma, UA also allowed an additional reduction in the volume of the myoma node by $27 \%$. 01/09/2014. Laparotomic myomiometrectomy without opening the uterine cavity, blood loss using the technique of atraumatic controlled vasocompression - up to $150 \mathrm{ml}$.

\section{Results}

The opening of the pseudocapsule of the uterine myoma has passed without difficulties (Figure 1). The absence of the effect of UA on pseudocapsule is a significant advantage of the drug compared to aHNRH, which often violates the boundary between the myomatous node and the pseudocapsule and causes difficulties in subsequent enucleation of the myomatous nodes. UA acted selectively, selectively suppressing proliferation and enhancing apoptosis, and also causing the ECM regression ${ }^{5}$ only in the leiomyoma, therefore, the surrounding myometrium after drug treatment was not changed (Figure 2), which allowed qualitatively to restore the uterine wall).

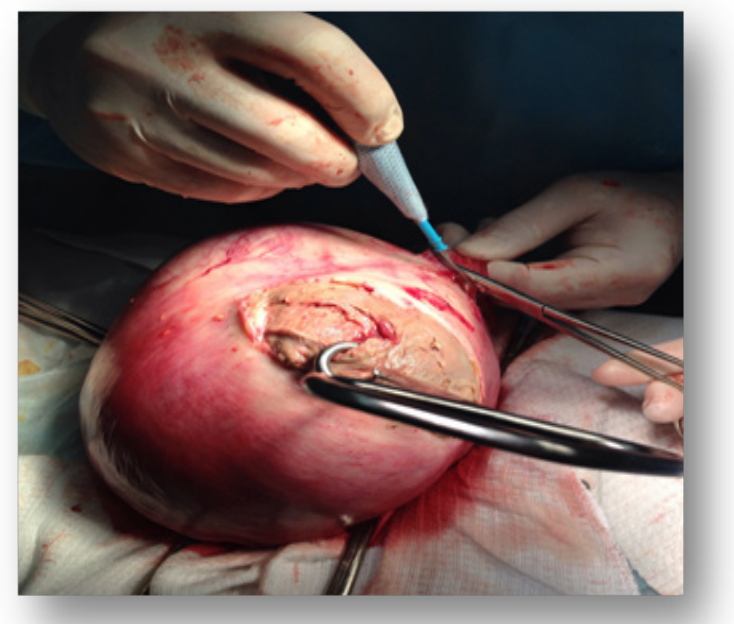

Figure I Opening of the pseudocapsule of the uterine myoma.

\section{The postoperative period proceeded without compli- cations}

01/12/2014. in satisfactory condition discharged from the hospital.

01/20/2014. Result of histological examination: 1 mitotically active leiomyoma with a site of unchanged myometrium and serosa. Leiomyoma is represented by sites that have different structures. Some sites have a structure of mitotically active leiomyoma (up to 9 mitoses in 10 fields of vision, $\mathrm{x} 400$ ), but without atypical mitoses or atypia of leiomyocytes, foci of necrosis. In addition to mitosis, there are also many leiomyocytes in the apoptosis stage. The pseudocapsule is expressed.

Appointed Buserelin Depot $3.75 \mathrm{mg}$ for 1 in / m injection, starting with the 1 st day of regular menstruation once in 28 days number 3 . 
05/19/2014. Ultrasound: uterus $4.9 \times 4.9 \times 5.6 \mathrm{~cm}$; M-echo $3 \mathrm{~mm}$, endometrium linear, the contours of the uterine cavity are even, clear. In the ovaries there are 5 follicles in the cut.

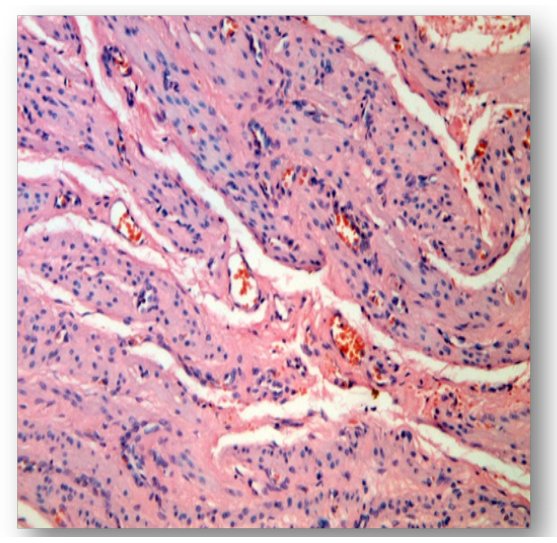

Figure 2 Myometrium surrounding the node of the leiomyoma. The structure is not changed, perivascular growth zones are absent, acute vasoconstriction. Stained with hematoxylin and eosin, $x 250$.

$08 / 20 / 2014$. To restore menstrual function, COC is prescribed Siluet in connection with the reproductive plans of the patient in 2015.

Since January 2015, COC has been discontinued, the last menstruation is 30.12. 2014-02.01. 2015., on time, of a moderate nature. Reproductive plans. Spermogramm - N. At this stage, it was recommended to track ovulation and the usefulness of the second phase of the menstrual cycle and realize the reproductive function.

10.02.15. Delayed menstruation for 11 days - the pregnancy test is positive.

15.02.15. Delayed menstruation for 16days - pregnancy test positive

26.02.15. Ultrasound - progressive uterine pregnancy 6weeks.

15.05.15. Ultrasound is a normally progressive pregnancy of 17 weeks.

Following a US examination at $34 \mathrm{GW}$ : Normally progressing pregnancy.

At 38-39 GW: Successful delivery through C-section with live male fetus $(3,050 \mathrm{~g})$.

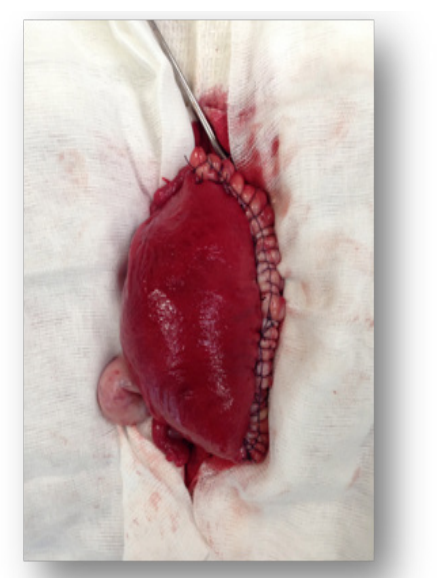

Figure 3 The wall of the uterus is restored by 3 -stranded sutures.

\section{Discussion}

Optimal treatment for patients with uterine myoma who are planning a pregnancy is not definitively determined. ${ }^{6-9}$ It was reported that myomectomy can improve reproductive function in women with submucosa and intra-wall fibroids. ${ }^{6}$ Nevertheless, to determine the effect of myomectomy on improving reproductive function, data obtained in randomized controlled trials are still insufficient. ${ }^{8}$ On the other hand, the currently available data are insufficient to determine whether radiological procedures are suitable for the treatment of women with non-asymptomatic fibroids who wish to maintain reproductive function. ${ }^{7}$ Alternative methods of drug treatment have limitations ${ }^{6}$ and are not recognized as reliable, ensuring the preservation of reproductive function. ${ }^{7}$ Treatment with $5 \mathrm{mg}$ of unacceptable acetate for 13 weeks prevents excessive bleeding and reduces the total volume of uterine fibroids. ${ }^{2,10}$

In our patient, the endometrial cavity was deformed by large interstitial myoma, which was accompanied by menorrhagia and anemia. Therefore, in order to preserve and restore the reproductive function of a young, nulliparous woman, we went on a long and welldeveloped path of preoperative preparation of such patients with the use of stage embolization of fibroids uterus.

\section{Conclusion}

In order to create conditions for organ-preserving treatment and reduce operational risks, a differentiated approach, sometimes combining medicamentous and invasive treatment, is needed. It may be advisable to use a selective modulator of progesterone receptors $\mathrm{UA}$ and $\mathrm{Fe}++-$ as an important step in the management of patients with uterine myoma.

\section{Acknowledgements}

A Tikhomirov has no relevant affiliations or financial involvement with any organisations or entity with financial interest in or financial conflict with the subject matter or materials discussed in the manuscript apart from those disclosed.

\section{Conflict of interest}

Author declares that there is no conflict of interest.

\section{References}

1. Bulun SE. Uterine fibroids. N Engl J Med. 2013;369(14):1344-1355.

2. Donnez J, Tomaszewski J, Vazquez F, et al. Ulipristal acetate versus leuprolide acetate for uterine fibroids. $N$ Engl J Med. 202;366(5):421-432.

3. Donnez J, Vazquez F, Tomaszewski J, et al. Long-term treatment of uterine fibroids with ulipristal acetate. Fertility and Sterility. 2014;101(6):1565. e1-1573.e18.

4. Williams AR, Bergeron C, Barlow DH, et al. Endometrial morphology after treatment of uterine fibroids with the selective progesterone receptor modulator, ulipristal acetate. International Journal of Gynecological Pathology. 2012;31(6):556-569.

5. Tikhomirov AL, Zayratyants OV. Inhibition of angiogenesis and growth factor with induction of metalloproteinase activity in the regression of uterine leiomyomas after Esmya treatment course. Obstetrics and Gynecology. 2014;36(6):590-595.

6. Donnez J, Tatarchuk TF, Bouchard P, et al. Ulipristal acetate versus placebo for fibroid treatment before surgery. N Engl J Med. 2012;366(5):409-420. 
7. Kroon B, Johnson N, Chapman M, et al. Fibroids in infertility - consensus statement from ACCEPT (Australasian CREI Consensus Expert Panel on Trial Evidence). Aust N Z J Obstet Gynaecol. 2011;51(4):289-295.

8. Metwally M, Cheong YC, Horne AW. Surgical treatment of fibroids for subfertility. Cochrane Database of Systematic Reviews. 2012;11:ID CD003857.
9. http://www.medicines.org.uk/EMC/medicine/26068/SPC/Es mya $+5+\mathrm{mg}+$ Tablets $+($ ulipristal + acetate $)$

10. Wdowiak A. Pre-treatment with ulipristal acetate before ICSI procedure: a case report. Przeglad Menopauzalnyy. 2013;6:496-500. 